\title{
Robust Dual Motion Deblurring *
}

\author{
Jia Chen Lu Yuan Chi-Keung Tang Long Quan \\ Vision and Graphics Group \\ The Hong Kong University of Science and Technology \\ \{jiachen, luyuan, cktang, quan\}@cse.ust.hk
}

\begin{abstract}
This paper presents a robust algorithm to deblur two consecutively captured blurred photos from camera shaking. Previous dual motion deblurring algorithms succeeded in small and simple motion blur and are very sensitive to noise. We develop a robust feedback algorithm to perform iteratively kernel estimation and image deblurring. In kernel estimation, the stability and capability of the algorithm is greatly improved by incorporating a robust cost function and a set of kernel priors. The robust cost function serves to reject outliers and noise, while kernel priors, including sparseness and continuity, remove ambiguity and maintain kernel shape. In deblurring, we propose a novel and robust approach which takes two blurred images as input to infer the clear image. The deblurred image is then used as feedback to refine kernel estimation. Our method can successfully estimate large and complex motion blurs which cannot be handled by previous dual or single image motion deblurring algorithms. The results are shown to be significantly better than those of previous approaches.
\end{abstract}

\section{Introduction}

Motion deblurring is a highly ill-posed problem where the observed blurred image $B$ is the convolution of unknown latent image $I$ with an unknown blur kernel $\mathbf{k}$, plus noise $n$ :

$$
B=I \otimes \mathbf{k}+n .
$$

Deblurring a single image is difficult when the kernel is unknown. Blind deconvolution works only for low frequency blur kernels. Image prior is used to estimate the blur kernel from real images $[5,10]$. However, these approaches are not robust enough and sometimes heavy human interactions are involved [8].

Methods using multiple images to perform blur estimation have been proposed in $[1,4,13,15]$. They seek

\footnotetext{
* This research is supported by the Research Grant Council of Hong Kong SAR, China: HKUST620207 and HKUST619006.
}
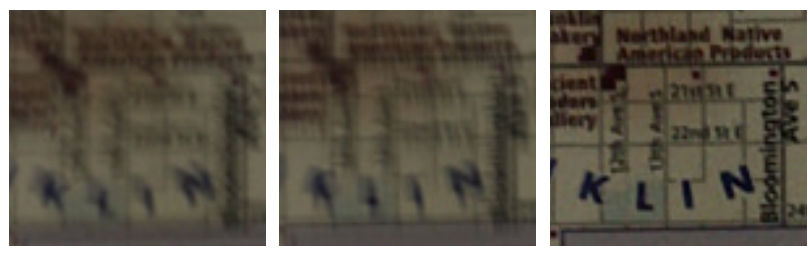

Figure 1. Dual-image deblurring given two blurred images. Full images are shown in Figure 8.

to utilize the correlation among blurred images, based on the assumption that all blur observations come from the same latent image. Promising results have been obtained using multiple-image deblurring algorithms but they are still limited to simple directional motion. Algorithm using blur/noisy image pair [18] yields good kernel estimation and deblurring results, but it needs an exposure bracketing function which is not available in many consumer level cameras, thus limiting the application range.

In this paper, we propose a dual-image deblurring algorithm which takes the advantage of burst capture capability of both compact and DSLR cameras. In kernel estimation, we propose a robust cost function to reject noise and outliers. We also apply a continuity prior together with sparseness prior to maintain kernel shape and resolve ambiguity. We will show that the estimation of blur kernels is greatly improved. After the motion kernels have been estimated, we propose a novel deblurring algorithm, which uses two blurred images to produce a clear image. The deblurring algorithm is robust to both kernel noise and image noise. It also greatly suppresses ringing artifacts while preserving image details. The estimation of blur kernels are further refined given the deblurred image in the iterative feedback procedure.

Because the proposed dual-image kernel estimation and dual-image deblurring modules are both robust, their combination will compensate each other to improve the accuracy. The feedback iteration robustly corrects estimation errors and gradually recovers image and kernel details. We will show by synthetic and real examples that the our approach greatly outperform previous approaches. 
This paper is organized as follows: We start in Section 2 by reviewing and analyzing previous dual image deblurring framework [13]. After we have analyzed the limitations of previous work, we present the robust kernel estimation algorithm in Section 3. We then describe the dual-image deblurring algorithm in Section 4. The feedback approach is then presented in Section 5 and our results are shown in Section 6. This paper is concluded in Section 7.

\section{Analysis of Previous Work}

In this section we briefly review and analyze previous dual image kernel estimation algorithm [13]. Assuming the blurring process for two observations $B_{1}$ and $B_{2}$ can be written as follows:

$$
B_{1}=I \otimes \mathbf{k}_{1}+n_{1}, \quad B_{2}=I \otimes \mathbf{k}_{2}+n_{2},
$$

where $I$ is the latent clear image. $\mathbf{k}_{1}, \mathbf{k}_{2}$ are two motion blur kernels to be solved by minimizing the following energy function:

$$
E\left(\mathbf{k}_{1}, \mathbf{k}_{2}\right)=E_{d}\left(\mathbf{k}_{1}, \mathbf{k}_{2}\right)+\alpha\left(E_{r e g}\left(\mathbf{k}_{1}\right)+E_{r e g}\left(\mathbf{k}_{2}\right)\right),
$$

where the data energy

$$
E_{d}\left(\mathbf{k}_{1}, \mathbf{k}_{2}\right)=\left\|B_{1} \otimes \mathbf{k}_{2}-B_{2} \otimes \mathbf{k}_{1}\right\|^{2},
$$

and regularization $E_{\text {reg }}\left(\mathbf{k}_{i}\right)=\left\|\mathbf{k}_{i}\right\|^{2}$ are both quadratic. Although good results have been shown in previous papers, this scheme is very limited.

First of all, quadratic cost function works well only when the estimation noise is Gaussian. In the data energy, the estimation noise is non-Gaussian even we assume the original observation noise is Gaussian. We substitute Equation 1 into the error term defined in the data energy and we compute the estimation noise

$$
\begin{aligned}
n_{\text {est }} & =B_{1} \otimes \mathbf{k}_{2}-B_{2} \otimes \mathbf{k}_{1} \\
& =n_{1} \otimes \mathbf{k}_{2}-n_{2} \otimes \mathbf{k}_{1},
\end{aligned}
$$

where it can be easily seen that the actual estimation noise associated with the blur process is highly non-Gaussian.

The second limitation of the formulation is that the solution is ambiguous. Suppose $\left(\mathbf{k}_{1}, \mathbf{k}_{2}\right)$ is the correct solution, then all motion kernel pairs $\left(\mathbf{k}_{1} \otimes \mathbf{k}^{\prime}, \mathbf{k}_{2} \otimes \mathbf{k}^{\prime}\right)$ also minimize the data energy $E_{d}$ as long as the common blur kernel $\mathbf{k}^{\prime}$ is non-negative. Although the regularity term $E_{\text {reg }}$ helps to smooth kernel values in order to reduce noise, it does not help to resolve the ambiguity, because convolving one common kernel $\mathbf{k}^{\prime}$ may even reduce $E_{r e g}$ while keeping $E_{d}$ unchanged.

The reason why $[4,13]$ achieve good results despite the limitations we describe above is that they mainly deal with directional motion, which is relatively simple and restrains

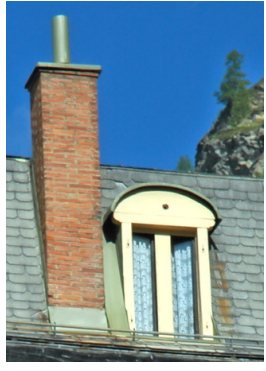

(a)

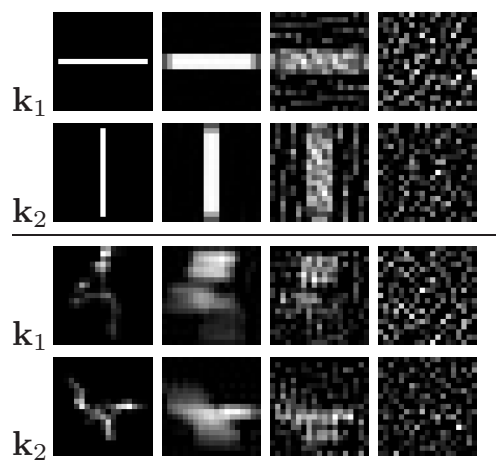

(b) (e) (c)

(d)
Figure 2. Kernel estimation under two pairs of synthetic motion. (a) Clear image. (b) Two pairs of ground truth kernels. (c)-(e) Estimation results at different noise levels. (c) $\sigma=0.0001$. (d) $\sigma=0.001$. (e) $\sigma=0.01$. We assume the range of image intensity to be $[0,1]$.

the ambiguity. We will show, by synthetic examples, that line motion can be easily resolved in noise-free situation. But this method is not robust when there exists observation noise, or the motion blur is complex.

As shown in the first two rows of Figure 2(c), the estimated line motion are close to the ground truth. If we use realistic complex blur kernels (resized from [5]) to synthesize motion blur, the results are very ambiguous as shown in the third and fourth rows. Furthermore, if noise $n_{1}, n_{2} \sim N\left(0, \sigma^{2}\right)$ is added to the observations $B_{1}$ and $B_{2}$, the estimation fails quickly when the noise level increases.

We analyze the reason why traditional approaches are adversely affected by noise, by examining the quadratic data energy of Equation 2 which is equivalent to $E(k)=$

\begin{tabular}{|l|l|l|l|l|l|l|}
\hline$\sigma$ & 0.0001 & 0.0005 & 0.001 & 0.005 & 0.01 & 0.02 \\
\hline$e$ & 0.0362 & 0.0560 & 0.1176 & 2.068 & 8.168 & 32.81 \\
\hline
\end{tabular}

Table 1. The minimum eigenvalue $e$ of $A^{T} A+\alpha^{2} I$ increases as the noise level $\sigma$ increases. We use the image and simple line motion kernel in Figure 2 and set $\alpha^{2}=0.03$.

$\mathbf{k}^{T}\left(A^{T} A+\alpha^{2} I\right) \mathbf{k}^{T}$ where $A=\left[A_{2},-A_{1}\right]$ is the combination of Toeplitz matrices and $\mathbf{k}=\left[\mathbf{k}_{1}, \mathbf{k}_{2}\right]$. Ideally, the solution is the eigenvector (with a scale/shift) corresponding to the minimum eigenvalue which ideally equals to $\alpha^{2}$. We compute the minimum eigenvalue of matrix $A^{T} A+\alpha^{2} I$ at different noise levels. It can be seen in Table 2, with the increase of noise level, the minimum eigenvalue also increases. In other words, the existence of noise affects the optimality of the correct solution, that is, making the estimation very vulnerable to local minima, as shown in Figure 2. 


\section{Robust Kernel Estimation}

Observation noise and outliers are important issues in motion deblurring when we deal with real-world images. In this section, we introduce a robust approach to estimate blur kernels. We propose to use a robust cost function as the estimator, which effectively rejects noise and outliers. Besides incorporating kernel sparseness prior to resolve ambiguity, we also propose a kernel continuity prior to increase the estimation robustness.

\subsection{Robust Cost Function}

We have shown that the quadratic cost function is not robust to non-Gaussian noise and we propose to use a more robust cost function. Robust statistics has been applied to a number of vision problems [2, 6, 9], where robust estimators has been proposed which are less sensitive to noise and outliers. In this work, we use the Lorentzian estimator [3]:

$$
\rho(r)=\log \left(1+\frac{1}{2}\left(\frac{r}{\varepsilon}\right)^{2}\right)
$$

The quadratic cost function and the robust cost function are compared in Figure 3. When the error is small, the cost of robust estimator grows faster than quadratic, and when the error is large, it increases at a pace slower than linear.

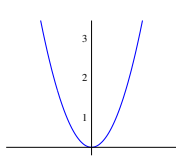

(a)

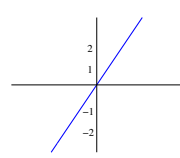

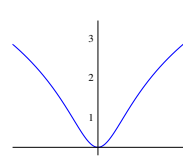

(b)

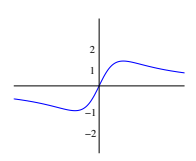

Figure 3. Comparing the quadratic and Lorentzian cost functions and their derivative functions. (a) Quadratic. (b) Lorentzian.

We then define the data energy of the dual deblur problem as follows:

$$
E_{d}\left(\mathbf{k}_{1}, \mathbf{k}_{2}\right)=\int_{\Omega} \rho\left(B_{1} \otimes \mathbf{k}_{2}-B_{2} \otimes \mathbf{k}_{1}\right) d \Omega,
$$

where $\rho(\cdot)$ is the robust cost function defined above.

In our implementation, we use an iterative reweighted least square (IRLS) approach to approximate the robust cost function. The residual comes from the data energy $r=A \mathbf{k}$. We define the diagonal reweighting matrix $W$ with elements

$$
w_{i i}=\frac{2}{2 \varepsilon^{2}+r_{i}^{2}} .
$$

The reweighted data energy now becomes $E_{d}(\mathbf{k})=$ $\mathbf{k}^{T} A^{T} W A \mathbf{k}$. Signals which are predicted accurately will be given larger weights in the next iteration, while the weights of outliers will be reduced. This procedure effectively overcomes noise and outliers, and will be evaluated in Section 3.3 .

\subsection{Kernel Prior: Sparseness and Continuity}

While the robust cost function improves the data energy in estimating blur kernel, we should also impose better kernel regularities in kernel estimation. It is well known that the kernel should be sparse, i.e., there are only a few large values in the kernel, while most values are zero. The sparseness prior, as described in [5], can be formulated by fitting a mixture of exponential distributions on the kernel values:

$$
p\left(k^{j}\right) \propto \sum_{m} w_{m} \lambda_{m} e^{-\lambda_{m} k^{j}}, 0<k^{j}<1
$$

and the sparseness energy defined on kernel $\mathbf{k}_{i}$ can be written as:

$$
E_{s}\left(\mathbf{k}_{i}\right)=-\alpha \sum_{j}\left(\log p\left(k_{i}^{j}\right)\right)
$$

The sparseness prior effectively prevents the kernel from being too smooth and can be implemented by IRLS using similar techniques in [11]. However, another problem arises when using sparseness prior: the estimated kernel sometimes becomes too sparse with only a few isolated dots, as shown in Figure 4.

One important observation of the motion blur is that the motion is continuous, which reflects the fact that the CCD sensors are continuously charged during camera shaking. It is therefore desirable that the value of the blur kernel is spatially smooth.

We propose a kernel continuity prior to constrain the spatial smoothness of kernel in shape. It is defined by the anisotropic diffusion tensor where we treat the kernel $\mathrm{k}$ as a $2 \mathrm{D}$ image,

$$
\mathbf{D}=\frac{\nabla \mathbf{k}^{\perp} \nabla \mathbf{k}^{\perp^{T}}}{\|\nabla \mathbf{k}\|},
$$

where $\nabla \mathbf{k}^{\perp}$ is the vector perpendicular to the local gradient $\nabla \mathbf{k}$. The energy which regularizes the kernel continuity is thus defined as:

$$
E_{c}(\mathbf{k})=\int_{\Omega} \nabla \mathbf{k}^{T} \mathbf{D}(\nabla \mathbf{k}) \nabla \mathbf{k} d \Omega
$$

Note that it differs from the regularization term $E_{r e g}(\mathbf{k})$, which only smoothes the values of the kernel elements. The anisotropic diffusion tensor has a nice property, where the amount of diffusion depends on local geometry. As described in $[16,17]$, the diffusion can be implemented by a local 2D Gaussian convolution at each iteration. The size and orientation of Gaussian convolution are adaptive to local structure of the blur kernel at the current iteration.

The total energy of kernel estimation is summarized as:

$$
E\left(\mathbf{k}_{1}, \mathbf{k}_{2}\right)=E_{d}\left(\mathbf{k}_{1}, \mathbf{k}_{2}\right)+\sum_{i=1}^{2}\left(E_{s}\left(\mathbf{k}_{i}\right)+E_{c}\left(\mathbf{k}_{i}\right)\right),
$$

and it is minimized by iteratively applying the conjugate gradient updates for the data and sparseness energy and the anisotropic diffusion. This process converges usually within 50 iterations. 


\subsection{Evaluation}

We summarize this section by evaluating the proposed kernel estimation approach. As shown in Figure 4. Using sparseness prior with quadratic cost gives good estimation at medium noise level but fails at higher noise level. Combining the robust cost function and kernel continuity prior with sparseness, kernels can be well estimated at very high noise level.

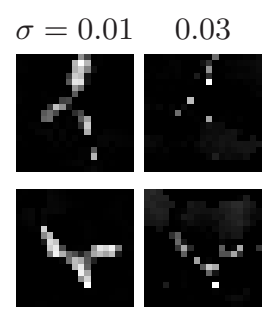

(a)

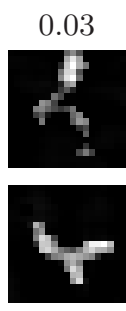

(b)

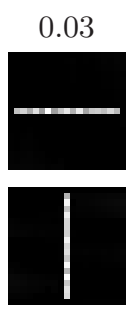

(c)

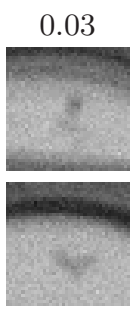

(d)
0.03
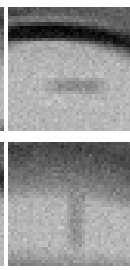

(e)
Figure 4. Evaluation against noise. (a) Using sparseness prior and quadratic cost function, at two different noise levels. (b) and (c) Estimation using robust cost function, sparseness and continuity priors. Please refer to Figure 2 for the ground truth kernels. (d) and (e) Noisy patches cropped from the grayscale blurred images.

We further test the robust data energy against outliers. We occlude the clear image with two different occluders before applying motion blur, as shown in Figure 5. The proposed kernel estimation algorithm automatically downgrades the weight of outliers thanks to the robust cost function. As shown by the estimated kernels, the proposed robust kernel estimation algorithm is not sensitive to outliers.

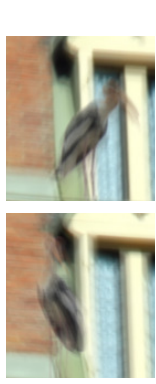

(a)

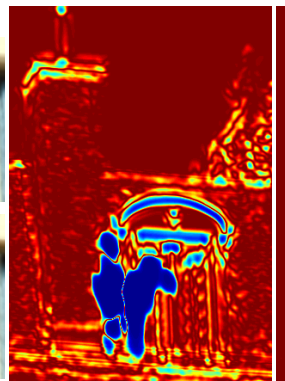

(b)

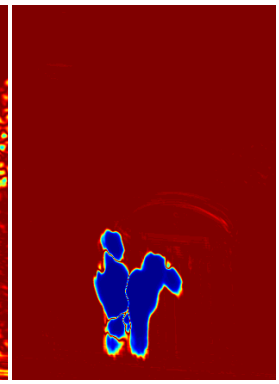

(c)

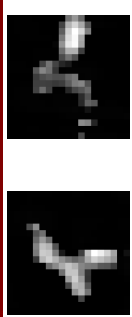

(d)
Figure 5. Robust outlier rejection. (a) Two blurred images are occluded differently. (b) Weight map of the second IRLS iteration. (c) Final weight map. (d) Estimated kernels.

\section{Dual-image Deblurring}

One of the most robust 2D deconvolution techniques is the Richardson-Lucy (RL) algorithm [12, 14], which computes the maximum likelihood estimate of the clear image. However, it usually generates ringing artifacts in the deblurred image. In [11], a deconvolution algorithm using natural image prior is introduced to reduce ringing ar- tifacts providing accurate blur kernel. The blurred/noisy approach [18] deblurs image using gain controlled RL algorithm to suppress ringing. Because we take two blurred images as input, our approach is different.

We use the estimated blur kernels together with two blurred images to reconstruct the clear image. In this dualimage deblurring algorithm, a deconvolution energy is defined:

$E(I)=\rho_{1}\left(I \otimes \mathbf{k}_{1}-B_{1}\right)+\rho_{2}\left(I \otimes \mathbf{k}_{2}-B_{2}\right)+E_{s}(I),(12$

where $E_{s}(I)$ is the sparseness prior of image gradients as used in [11]. One important advantage of our approach is that we make use of the strong constraint that the deblurred image should be consistent with both blurred observations. However, there might exist outliers or inconsistence between two images. In order to deal with this, we design the correlated robust cost functions $\rho_{1}(\cdot), \rho_{2}(\cdot)$ which have similar property as that we used in kernel estimation.

In dual-image deblurring, outliers are the set of pixels inconsistent between two blurred images, such as the two artificially added birds in Figure 5. We should first identify outliers and avoid inconsistent solution by selecting pixels to deblur from only one blurred image at these locations. We implement the robust cost functions by reweighted least square and correlate the weighting matrices $W$ and $W^{\prime}$. We rewrite Equation 12 as follows:

$E(I)=\left\|W\left(I \otimes \mathbf{k}_{1}-B_{1}\right)\right\|^{2}+\left\|W^{\prime}\left(I \otimes \mathbf{k}_{2}-B_{2}\right)\right\|^{2}+E_{s}(I)$.

We then define the residuals and uncorrelated weights:

$$
\begin{aligned}
& r=I \otimes \mathbf{k}_{1}-B_{1}, \quad v_{i}=\frac{2}{2 \varepsilon^{2}+r_{i}^{2}} \\
& r^{\prime}=I \otimes \mathbf{k}_{2}-B_{2}, \quad v_{i}^{\prime}=\frac{2}{2 \varepsilon^{2}+{r_{i}^{\prime}}^{2}} .
\end{aligned}
$$

The correlated reweight matrix $W$ and $W^{\prime}$ are diagonal with elements:

$$
\begin{aligned}
w_{i i} & =\left\{\begin{array}{ll}
\frac{v_{i}}{v_{i}+v_{i}^{\prime}}, v_{i} \geq \tau & \text { or } \quad v_{i}^{\prime} \geq \tau \\
P, & v_{i}<\tau \text { and } v_{i}^{\prime}<\tau
\end{array},\right. \\
w_{i i}^{\prime} & =1-w_{i i} .
\end{aligned}
$$

The threshold $\tau$ is set to reject outliers. When the weights $v$ and $v^{\prime}$ from either image is large, pixels from both images are used to reconstruct the clear image. At locations where the weights from both images are small, the algorithm switches to single image deconvolution. $P=\{0,1\}$ is a predefined indicator to decide which image the algorithm relies on when outlier/inconsistence is detected.

We use the blurred images and estimated kernels from Figure 5 to perform deblurring. As shown in Figure 6, our algorithm improves significantly over single-image RL method [12] and algorithm using sparseness prior [11]. 
They exhibit either visible ringing artifacts, or loss of details. On the contrary, there are much fewer artifacts and details are well retained in our result. We also compute the $P S N R$ s of the deblurred images, and the dual-image approach has much higher PSNR than other two methods.

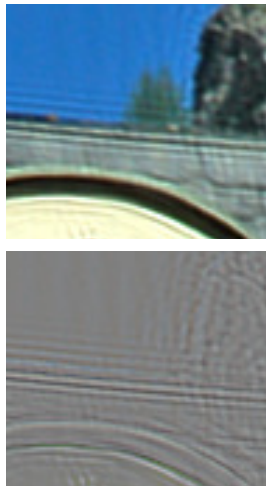

Single image RL

(a) $26.14 d B$

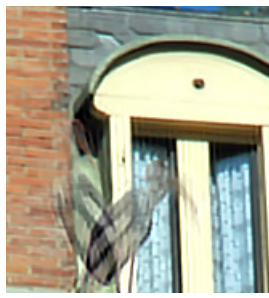

$\mathrm{W} / \mathrm{O}$ rejection

(d)
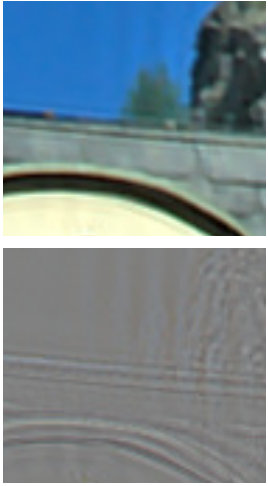

Single w/ prior

(b) $30.30 d B$

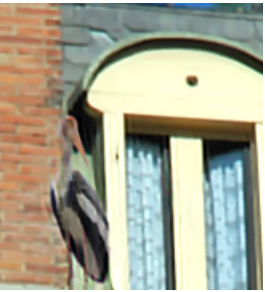

With outlier rejection

(e)

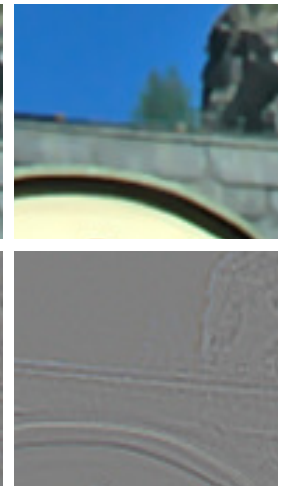

Dual-image w/ prior (c) $32.64 d B$

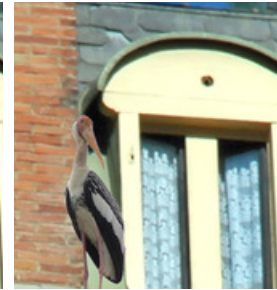

Ground truth

(f)
Figure 6. Deblurring synthetic images. (a)-(c) compare deblurring result with previous approaches. The second row shows the residual comparing to the ground truth. (d)-(f) show the effectiveness of dual-image deblurring with outlier rejection.

\section{Robust Feedback Algorithm for Joint Ker- nel Estimation and Image Deblurring}

We have presented robust kernel estimation and deblurring algorithms in previous sections. As we have demonstrated, our dual-image deblurring algorithm produces good results even when the estimated kernels are not perfectly accurate, because it can correct errors by enforcing consistence between two observations. We believe that the priors used in kernel estimation and those used in image deblurring provide different information and the deblurred image can be used to help the kernel estimation. In this section, we present a novel feedback approach to iteratively refine the kernel estimation as well as the deblurred images.

After we have obtained the clear image $I$ from Section 4, we feed it back to the kernel estimation step, where a feedback energy is defined as:

$$
E_{f}\left(\mathbf{k}_{i}\right)=\int_{\Omega} \rho\left(I \otimes \mathbf{k}_{i}-B_{i}\right) d \Omega .
$$

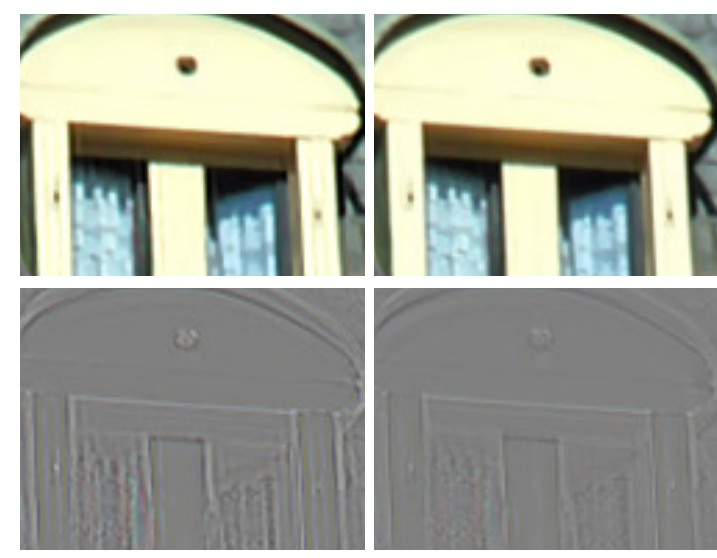

(a) $32.64 d B$

(b) $35.39 d B$

Figure 7. Deblurred result before and after feedback. The second row shows the residual comparing to the ground truth.

We use the robust cost function again, because it can also account for inaccurately estimated pixels values in the deblurred image $I$ which can be suppressed as outliers.

The feedback energy is combined with the original data energy and kernel priors. Now we write the kernel estimation energy as:

$$
\begin{aligned}
E\left(\mathbf{k}_{1}, \mathbf{k}_{2}\right) & =E_{d}\left(\mathbf{k}_{1}, \mathbf{k}_{2}\right)+\sum_{i} E_{f}\left(\mathbf{k}_{i}\right) \\
& +\sum_{i}\left(\alpha_{s} E_{s}\left(\mathbf{k}_{i}\right)+\alpha_{c} E_{c}\left(\mathbf{k}_{i}\right)\right), i=1,2 .
\end{aligned}
$$

We now summarize the proposed feedback approach as in Algorithm 1.

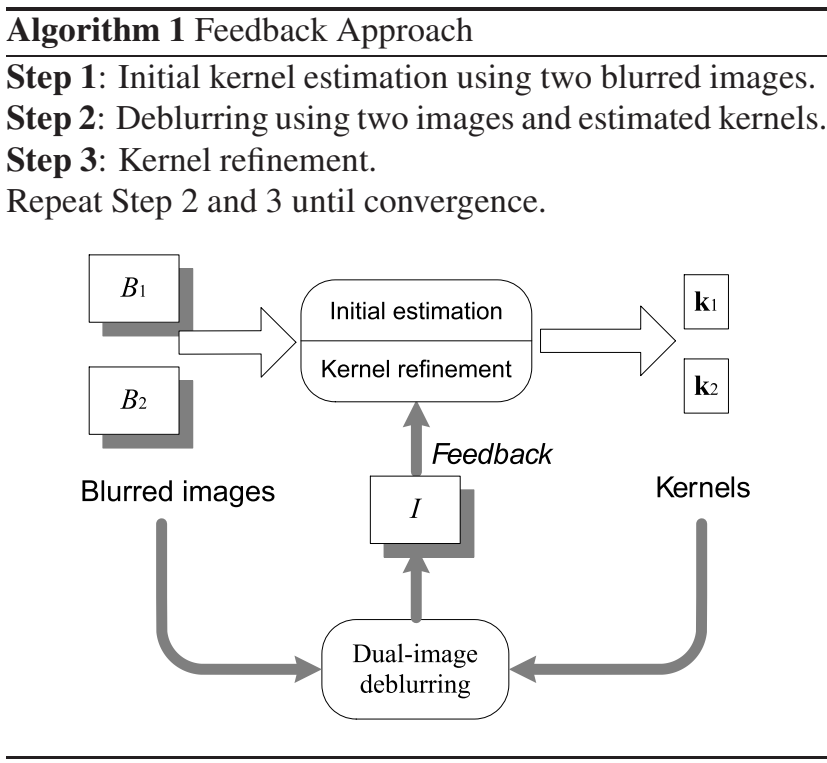

To illustrate the effectiveness of the feedback algorithm, we compare the close-up views of the deblurred results before and after executing the feedback iterations. The visual 

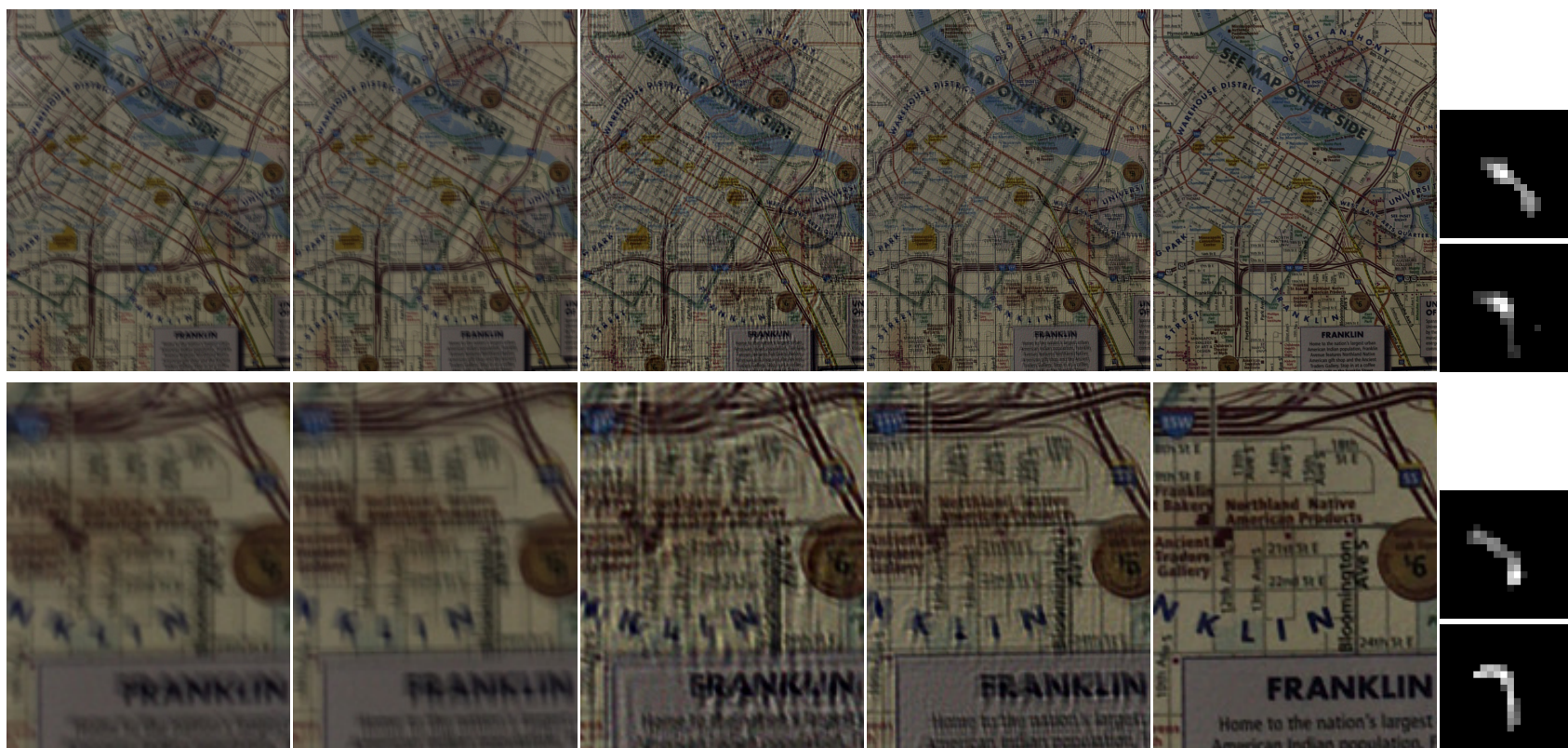

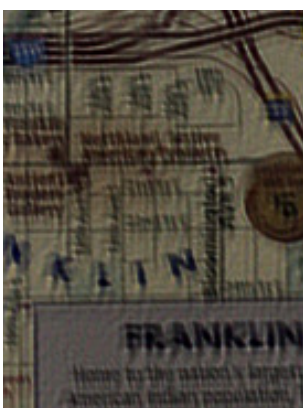

(b)

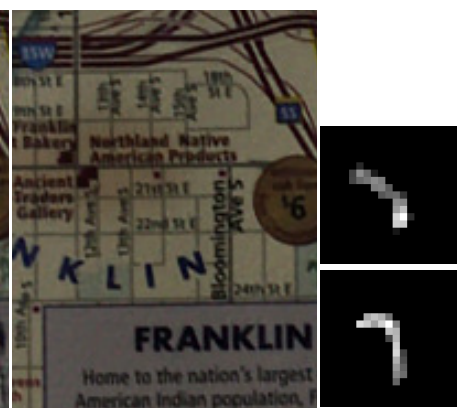

(c)

(d)

Figure 8. Deblurring MAP photos. (a) Two blurred inputs. (b) Deblurred results of (a), using single image kernel estimation [5] and RL deblurring algorithm. (c) Deblurred image using the proposed approach. (d) Top: estimated kernels from two blurred images in (a) respectively using single image kernel estimation. Bottom: estimated kernels using proposed approach. Kernel size is 19 by 19.

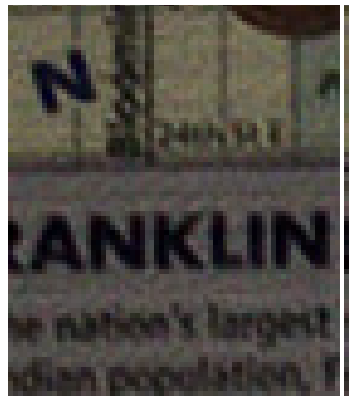

(a)

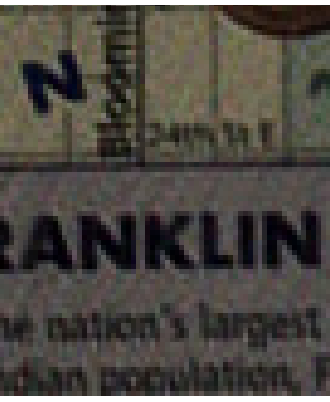

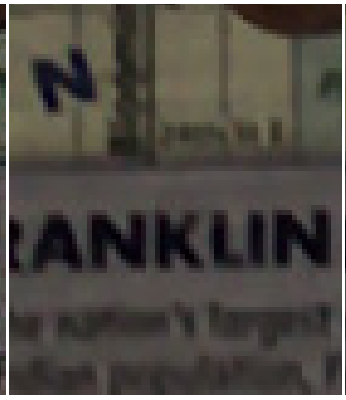

(b)

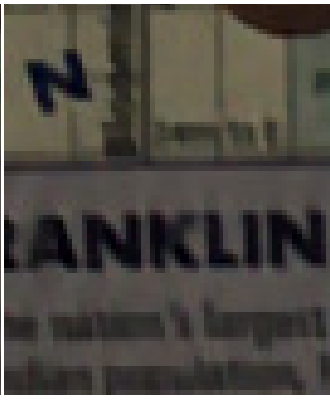

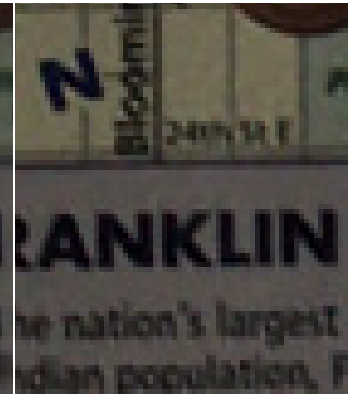

(c)

Figure 9. Comparing deblurring algorithms using the estimated kernels from our approach. (a) Single image deblurring results using RL algorithm. (b) Single image deblurring results using image priors [11]. (c) Dual-image deblurring results.

artifacts are further reduced after feedback. The increasing PSNR indicates that it improves the image accuracy as well as the kernel accuracy. We will show in Section 6, where the feedback approach refines kernel estimation and deblurring for very challenging real example.

\section{Experiments}

We have tested our algorithm on synthetic data and now we carry out experiments on a variety of real objects. The photos were taken by consecutive shooting at the same exposure time. We interactively align the blurred images using Photoshop [7] to rectify rotation and large translation between image pair.

The first example is MAP shown in Figure 8. We com- pare kernel estimation with single image algorithm. Two blur kernels are independently estimated as described in [5]. We then deblur the two input images using the RL algorithm. We can see that the single image approach estimates reasonable kernels but they are not accurate enough. The kernels estimated from the proposed approach are much more accurate as shown in the figure. The deblurred image from our dual-image deblurring algorithm is much better than that from the single image approach.

Using the MAP images, we also compare the deblurring results using the proposed dual-image algorithm with previous single image algorithms, where the same kernels estimated from our approach are used. From the close-up views in Figure 9, we can see that RL algorithm still produces visible ringing artifacts, even if the kernel is very accurate. The 


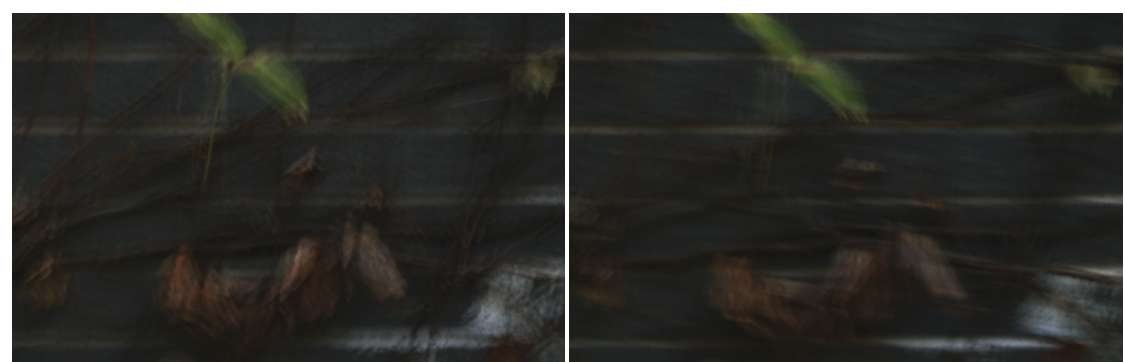

(a)
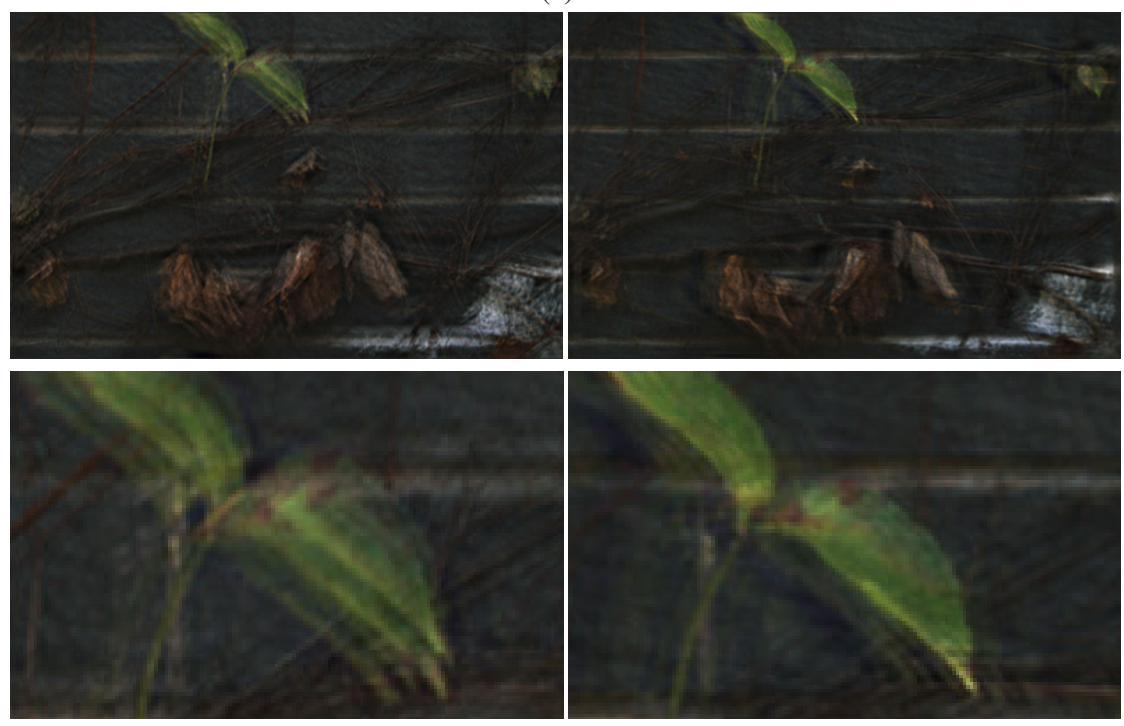

(c)
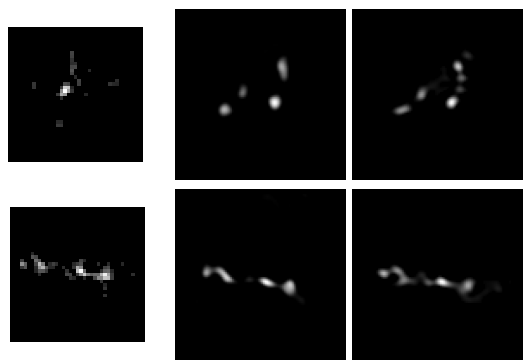

(b)
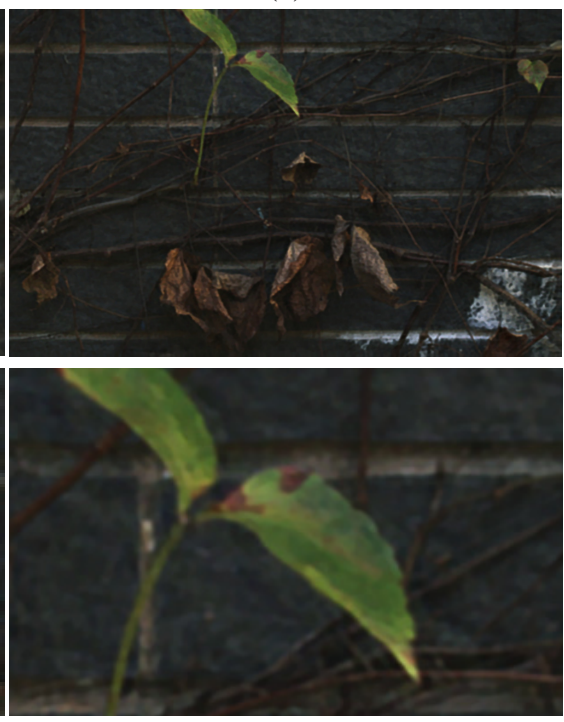

(d)

Figure 10. Deblurring WALL photos. (a) Two blurred inputs. (b) Left: estimated kernels using single image approach. Kernel size 51 by 51. Middle: estimated kernels using proposed approach, before feedback iterations. Right: refined kernels after feedback iterations. Kernel size is 99 by 99. (c) Deblurred images using kernels estimated from single image approach and RL deblurring algorithm. The input images are downsampled to $50 \%$ size. (d) Deblurred image using the proposed approach.

reason is that there exist image noise and loss of high frequency detail in both image and kernel. Using image priors in single-image deblurring can reduce artifacts but this method may oversmooth the image. Our result exhibits few artifact and preserves details very well, as shown in Figure 9(c), because the proposed approach successfully makes two input images complementary to each other.

We show one challenging example WALL in Figure 10. The blurred images are very noisy and the motion blur is quite large ( $50 \sim 75$ pixels $)$. Because the noise model in [5] may not afford to work at the original resolution, we downsample the input images to half resolution and run the single image algorithm to estimate blur kernels, which is suggested in the implementation of [5]. We tried our best to choose image region and tune parameters but the output kernel estimates seem not good enough. On the contrary, we runs the proposed approach at the original resolution, and it outputs much better kernels given two blurred images. Our approach robustly estimates good kernels in the initial iteration and they are refined after several feedback iterations.
Without the feedback iterations, the kernels are not accurate enough. In the end, the dual-image approach successfully outputs very clear image as shown in Figure 10.

We finally test our algorithm on another very challenging example FLOWER. There are several difficulties: the input blurred photos have pixels closed to saturation; small depth variation of scene; the camera position is not strictly fixed. We show in Figure 11 that our approach can still output good kernel estimation and deblurring results despite of these difficulties. This example shows the robustness of the proposed approach against outliers and noise, and it can be applied to a wide range of real-world images.

The running time of our algorithm is linear with the image size, kernel size and the number of iterations. For a small size problem with image size 320X240, and kernel size $25 \mathrm{X} 25$, each feedback iteration takes 3 minutes on a Intel P4 3.2G desktop machine. It usually requires about 5 to 10 feedback iterations to converge depending on the difficulty of the input data. 

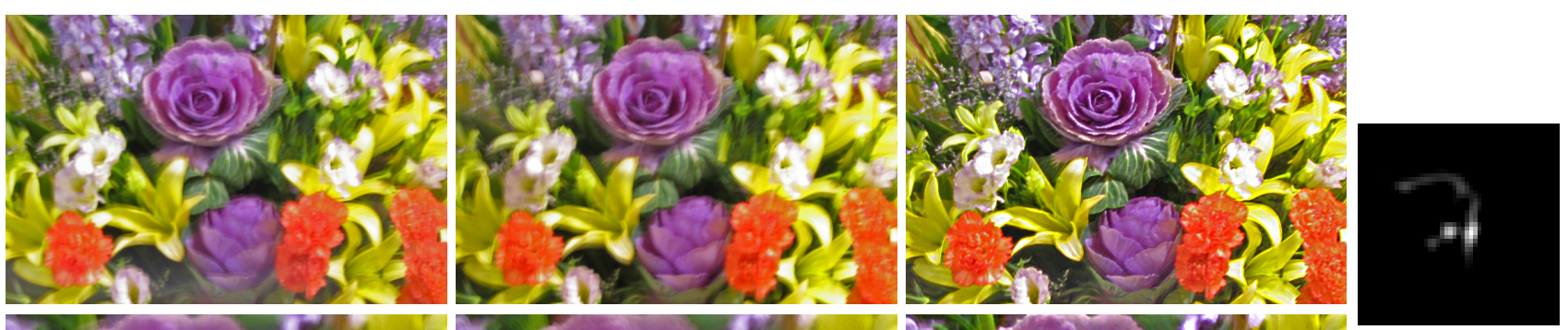
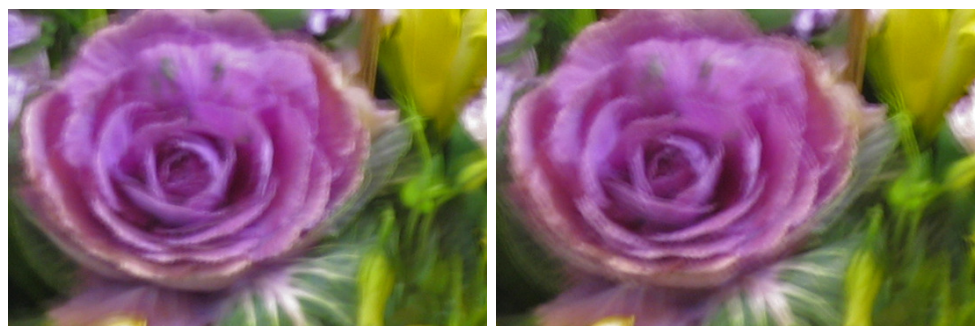

(a)

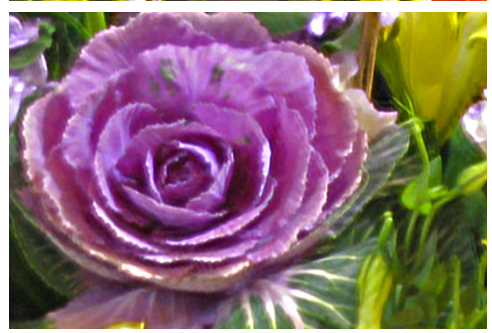

(b)

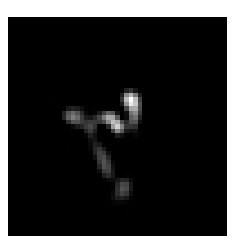

(c)

Figure 11. Deblurring FLOWER photos. (a) Two blurred inputs. (b) Deblurred result using the proposed approach. (c) Estimated kernels. Kernel size is 49 by 49 .

\section{Conclusions}

We have presented a novel dual-image motion deblurring algorithm. The individual modules, which include robust dual-image kernel estimation and dual-image deblurring algorithms outperform previous approaches respectively. The feedback approach proposed in this paper combines kernel estimation and deblurring to iteratively refine the results. We have shown by synthetic and real examples the effectiveness of our approach. Future work may include speeding up the system and investigating automatic alignment algorithms combined with deblurring, so that our approach can be applied to multiple input images and videos.

\section{References}

[1] B. Bascle, A. Blake, and A. Zisserman. Motion deblurring and super-resolution from an image sequence. In European Conference on Computer Vision (ECCV), pages 573-582, 1996.

[2] M. Black and A. Rangarajan. The unification of line processes, outlier rejection, and robust statistics with applications to early vision. International Journal of Computer Vision, 1996.

[3] M. J. Black and P. Anandan. A framework for the robust estimation of optical flow. In International Conference on Computer Vision (ICCV), 1993.

[4] S. Cho, Y. Matsushita, and S. Lee. Removing non-uniform motion blur from images. In International Conference on Computer Vision (ICCV), 2007.

[5] R. Fergus, B. Singh, A. Hertzmann, S. T. Roweis, and W. T. Freeman. Removing camera shake from a single image. ACM Trans. Graph. (SIGGRAPH), 25(3):787-794, 2006.

[6] P. J. Huber. Robust Statistics. Wiley, 1981.

[7] Adobe System Inc. Automatically align image layers. http://www.adobe.com/support/photoshop/, 2007.
[8] J. Jia. Single image motion deblurring using transparency. In IEEE Conference on Computer Vision and Pattern Recognition (CVPR), 2007.

[9] Q. Ke and T. Kanade. Robust $l_{1}$ norm factorization in the presence of outliers and missing data by alternative convex programming. In IEEE Conference on Computer Vision and Pattern Recognition (CVPR), 2005.

[10] A. Levin. Blind motion deblurring using image statistics. In NIPS, 2006.

[11] A. Levin, R. Fergus, F. Durand, and W. T. Freeman. Image and depth from a conventional camera with a coded aperture. ACM Trans. Graph. (SIGGRAPH), 26(3):70, 2007.

[12] L. B. Lucy. An iterative technique for the rectification of observed distributions. Astronomical Journal, 79:745, 1974.

[13] A. Rav-Acha and S. Peleg. Two motion blurred images are better than one. Pattern Recognition Letters, 26:311-317, 2005.

[14] W. H. Richardson. Bayesian-based iterative method of image restoration. Journal of The Optical Society of America, 62(1):55, 1972.

[15] Y.-W. Tai, H. Du, M. S. Brown, and S. Lin. Image/video deblurring using a hybrid camera. In IEEE Conference on Computer Vision and Pattern Recognition (CVPR), 2008.

[16] D. Tschumperle and R. Deriche. Vector-valued image regularization with pde's : A common framework for different applications. IEEE Transactions on Pattern Analysis and Machine Intelligence, 27(4):506, 2005.

[17] J. Xiao, H. Cheng, H. Sawhney, C. Rao, and M. Isnardi. Bilateral filtering-based optical flow estimation with occlusion detection. In European Conference on Computer Vision (ECCV), 2006.

[18] L. Yuan, J. Sun, L. Quan, and H.-Y. Shum. Image deblurring with blurred/noisy image pairs. ACM Trans. Graph. (SIGGRAPH), 26(3):1, 2007. 